\title{
Anmerkungen zur Debatte zwischen Schelling und Eschenmayer in den Jahren 1803-1804
}

\author{
Comments on the debate between Schelling and \\ Eschenmayer in the years 1803-1804
}

\author{
PATRICK LEISTNER \\ Evangelisch-Theologische Fakultät der Universität Wien (Alemania)
}

Recibido: 07-04-2011 Aceptado definitivamente: 02-05-2014

\begin{abstract}
ZUSAMMENFASSUNG
Im Rahmen des Artikels wird die Debatte zwischen Schelling und Eschenmayer in den Jahren 1803-1804 als eine Kontroverse analysiert, die deren vorherige um die Naturphilosophie weiterführt. Der Zentralgedanke Eschenmayers wird in dessen Theorie der »Potenzierung« gesehen. Auf dieser Grundlage entwickelt Eschenmayer 1803 eine Theorie der intellektuellen Anschauung, die seine Theorie des »Überganges« in methodischer Hinsicht tragen und dabei den Glauben in einer grundsätzlichen Bedeutung für das Wissen und die Philosophie wiedereinführen soll. Ferner soll damit die Generierung von Differenz in der absoluten Identität erklärbar sein. So erscheint die Debatte über den Zusammenhang von Philosophie und Religion von 1803 und 1804 zunächst als eine Debatte um die Methodik und die Theorie der Philosophie selbst. Die Analyse von Schellings Methodik, der »Konstruktion«, macht die tiefen Differenzen zwischen Eschenmayer und Schelling deutlich. In der Konsequenz hat auch die von Schelling zwischen 1802 und 1804 präsentierte Religionsphilosophie eine grundlegend andere Bedeutung als Eschenmayers.
\end{abstract}

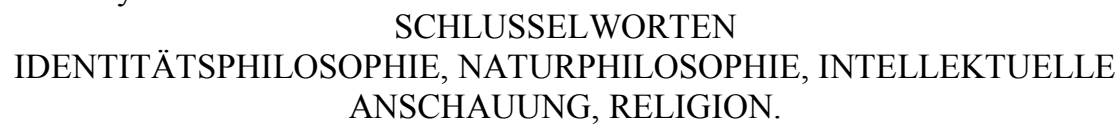

SCHLUSSELWORTEN

IDENTITÄTSPHILOSOPHIE, NATURPHILOSOPHIE, INTELLEKTUELLE ANSCHAUUNG, RELIGION. 


\begin{abstract}
Within the scope of this article the debate between Schelling and Eschenmayer in the years 1803-1804 is analysed as a controversy, that continuies the former concerning the philosophy of nature. The central thought of Eschenmayer in his theories of matter and also in his theorie of the philosophy proceeding to $\gg$ Nichtphilosophie «, is seen in the »Potenzierung «. On this basis he develops a theory of »Intellektuelle Anschauung" that should bear methodically his "progress in Nichtphilosophie« and reintroduce the religious belief in a fundamental meaning for the philosophy. Moreover it should explain the genesis of difference in the absolute identity. So the debate about the connection of philosophy and religion in 1803 and 1804 seems to be mainly one about the methods and theory of philosophy. Looking at Schellings method of »Konstruktion « the fundamental differences between both get visible. In the consequence Schellings philosophy of religion, that he presented between 1802 and 1804, has strictly another meaning than Eschenmayers.
\end{abstract}

\title{
KEY WORDS
}

philosophy of identity, philosophy of nature, intellectual intuition, religion.

IM ZUSAMMENHANG DES VORLIEGENDEN BANDES zu den aktuellen Folgen des Deutschen Idealismus könnte das Aufsatzthema zunächst ungewöhnlich wirken. Schellings Philosophie um 1800 mag vielerlei Wirkungen bis heute entfaltet haben, ja Gedanken Schellings mögen in der Gegenwart öfter anzutreffen sein als man denkt. Doch wird man die heute eher wenig erinnerte Debatte zwischen Friedrich Wilhelm Joseph Schelling und Adolph Carl August Eschenmayer auf den ersten Blick kaum zu denjenigen rechnen, die in der Gegenwart und für diese eine größere Bedeutung haben. Einer der Protagonisten, Eschenmayer, ist heute eine weitgehend unbekannte Gestalt der Philosophiegeschichte. Dasjenige, das die Streitsache für die Gegenwart jedoch interessant macht, ist, dass sie etwas Exemplarisches hat. Nicht nur ging es um eine moderne Bestimmung von Religion, sondern vor allem auch um das Verhältnis von Glauben und Wissen. Die Streitsache gehört in den Zusammenhang der großen und für die moderne Religionstheorie grundlegenden theologisch-religionsphilosophischen Streitsachen zwischen 1785 und 1811/12. ${ }^{1}$ Die Debatte von 1803 und 1804 eröffnet für die Theologie und Philosophie wichtige Perspektiven auf die methodischen Bedingungen einer Verhältnisbestimmung von Glauben und Wissen.

Ähnlich wie im Pantheismus- und Atheismusstreit zuvor ging es immer auch um grundsätzliche philosophische Fragen, insbesondere um das Verhältnis des Absoluten zum Endlichen, von Identität und Differenz. Einmal mehr war 1803

1 Vgl., hierzu Philosophisch-theologische Streitsachen. Pantheismusstreit- Atheismusstreit-Theismusstreit, hg. v. Christian Danz u. Georg Essen, Darmstadt 2012. 
und 1804 die Frage des »Überganges« Gegenstand einer Kontroverse, wobei diesmal die Identitätsphilosophie Schellings und ihr Absolutes von Eschenmayer im Hinblick auf diese Frage überprüft werden sollten. Auch wenn es in den Schriften der beiden Autoren zwischen 1803 und 1804 oft um abstrakte Grundfragen und Probleme der Identitätsphilosophie Schellings geht und die explizite Religionstheorie selbst relativ kurz kommt, so zeigen doch nicht zuletzt die Reflexionen Eschenmayers, wie sehr jene Themen von persönlicher und praktisch-religiöser Bedeutung waren.

Im Folgenden möchte ich zunächst Adolph Carl August Eschenmayer und seine Theorie der Nichtphilosophie in den Blick nehmen. Weniger soll Eschenmayers Theorie in den religionsphilosophischen Kontext um 1800 eingeordnet werden. Sondern eher soll dem eigenen Anspruch seiner Konzeption nachgegangen werden und die hinter der Theorie der Nichtphilosophie stehenden methodischen Vor-aussetzungen analysiert werden. (= I.) Die naturphilosophische Auseinandersetzung zwischen Schelling und Eschenmayer vor 1803 wird hier als Vorgeschichte der Debatte zwischen 1803 und 1804 in den Blick genommen. Danach wird auf Schelling eingegangen und einige Grundzüge seines Identitätssystems angesichts der von Eschenmayer seit 1801 formulierten kritischen Anfragen an dieses skizziert, um schlussendlich Schellings Zuordnung von Philosophie und Religion im Unterschied zu Eschenmayer zu rekonstruieren. (= II.)

\section{ESCHENMAYER UND DER ÜBERGANG DER PHILOSOPHIE IN NICHTPHILOSOPHIE}

Adolph Carl August Eschenmayer (1768-1852) ist außerhalb der Schellingforschung eine eher unbekannte Gestalt. Die Schellingforschung hat Eschenmayer zum Teil eine große Bedeutung für die Entwicklung des Werkes Schellings beigemessen. Die Streitsache rund um die Schrift Philosophie und Religion bzw. die Bearbeitung der Kritikpunkte, die Eschenmayer öffentlich an Schelling formulierte, hat etwa in der Sicht Hermann Zeltners die Weiterentwicklung der Identitätsphilosophie Schellings nach 1804 entscheidend angeregt. ${ }^{2}$ Setzt man die Bedeutung Eschenmayers für Schellings Werdegang geringer an, so lässt sich immer noch feststellen, dass Schelling durch Anfragen

2 Vgl., etwa Hermann Zeltner, Eschenmayer, Adolph Carl August, in: Neue Deutsche Biographie 4 (1959), S. 644: »Die Schrift: Die Philosophie in ihrem Übergang zur Nichtphilosophie (1803), in welcher er diese Lehre zuerst entwickelte, wurde für Schelling ein entscheidender Anstoß zur Weiterbildung seiner Lehre, wie überhaupt Eschenmayers bleibende Bedeutung in den Anregungen zu sehen ist, die andere Denker, unter ihnen zu wiederholten Malen Schelling, durch seinen aus wirklichem Mitdenken entsprungenen Widerspruch erfahren haben.« 
Eschenmayers über lange Jahre hinweg zu Stellungnahmen und Präzisierungen herausgefordert wurde.

Außerhalb der Schellingforschung wird Eschenmayer auch als Impulsgeber für die Entwicklung der modernen Psychiatrie angesehen. ${ }^{3}$ Die Theologie- und Philosophiegeschichte erwähnt ihn zum Teil jenseits der Kontroverse mit Schelling im Zusammenhang des Streits um das Leben Jesu von David Friedrich Strauß (1835/36), als Eschenmayer gegen diesen in der Schrift Der Ischariothismus unserer Tage von 1836 Stellung bezog. Letztlich richtete sich dies auch gegen die Philosophie Hegels, die in Eschenmayers Sicht Philosophie und Religion nicht angemessen unterschied. ${ }^{4}$ Strauß seinerseits, der Eschenmayers psychiatrische Vorlesungen in Tübingen besucht hatte ${ }^{5}$ und dessen Dissertation über die »Lehre von der »apokatastasis panton" in ihrer religionsgeschichtlichen Entwicklung « ${ }^{6} 1828$ von Eschenmayers Gutachten als mit dem Evangelium nicht vereinbar angesehen wurde, ${ }^{7}$ sparte dann auch nicht mit Polemik, wenn er, das heißt Strauß, Eschenmayer als »dilettantische[n] Philosoph[en]«, »unkritische[n] Systemspinner« und »düstere[n] religiöse[n] Fanatiker« bezeichnete. ${ }^{8}$

Eschenmayer war ein württembergischer Mediziner und Philosoph, der sich zunächst mit Naturphilosophie beschäftigte. Wie viele andere bedeutende Württemberger der Zeit studierte er zunächst an der Stuttgarter Karlsschule, wo ihn der Naturforscher Carl Friedrich Kielmeyer (1765-1844) prägte, der eine »Evolutionstheorie« der Natur entwickelte und die Natur als einen Organismus mit verschiedenen Grundkräfte verstand, der sich durch deren Gegeneinanderwirken entwickelt. ${ }^{9}$ Neben der Orientierung an Kielmeyers Theorie ist bei Eschenmayer das Interesse an der philosophischen Theorie der Natur, besonders

3 Vgl., Walter Wuttke, Materialien zu Leben und Werk Adolph Karl August von Eschenmayers, in: Sudhoffs Archiv. Zeitschrift für Wissenschaftsgeschichte, Bd. 56, Leipzig/ Wiesbaden 1972, S. 255-296, hier S. 261.

4 Vgl., etwa Adolph Carl August Eschenmayer, Der Ischariothismus unserer Tage. Eine Zugabe zu dem jüngst erschienenen Werke: Das Leben Jesu von Strauß, Teil 1, Tübingen 1835, S. VII.

5 Vgl., Wuttke, Materialien, S. 262.

6 Vgl., Theobald Ziegler, David Friedrich Strauß, Teil 1: 1808-1839, Strassburg 1908, S. 86.

7 Vgl., a.a.O., S. 85-91, hier S. 90.

8 So in David Friedrich Strauß, Justinus Kerner, in: Kleine Schriften, Neue Folge, Berlin 1866, S. 298-332, hier S. 320.

9 Vgl., Adolph Carl August Eschenmayer, Grundriß der Natur-Philosophie, Tübingen 1832, S. X: »Den ersten bleibenden Eindruk für Naturwissenschaft schöpfte ich aus den trefflichen Vorlesungen des Staatsraths Kielmeyer, zuerst Lehrer in der ehemaligen Academie in Stuttgart. Von ihm habe ich die Grundidee, nehmlich die in der lebenden Natur von der Pflanze bis zum Menschen beständig wechselnde Proportion der drei organischen Grundkräfte, die ich in dem Abschnitt der Biologie durchführte.« 
der Materie, zu erkennen, das bereits seine Dissertation von $1796^{10}$ zeigt. So konnte er sich schon früh der Naturphilosophie Schellings in ihrem Anliegen verbunden wissen, während auch Schelling die Arbeiten Eschenmayers lobend hervorhob und sich ein Briefwechsel zwischen beiden entwickelte. ${ }^{11}$

Eschenmayers Naturphilosophie ist eine umfassende Theorie der Natur und des Naturprozesses, in die naturwissenschaftliche Beobachtungen integriert sind. Hinter der von Eschenmayer angenommenen Möglichkeit, philosophisch »reine« Strukturen in der Beschreibung der Natur zu erfassen, steht seine Überzeugung, dass die apriorischen Bedingungen des Wissens zugleich die Bedingungen der Natur sind. ${ }^{12}$ In seinen ersten naturphilosophischen Schriften bestimmte er zudem das Verhältnis der induktiven empirischen Naturforschung, die »hypothetische Gesetzesannahmen « liefere, zur Deduktion von apriorischen Naturgesetzen aus höchsten Prinzipienals ein »Ergänzungsverhältnis «. ${ }^{13}$ Programmatisch ist etwa der Titel einer Schrift aus dem Jahr 1797: Versuch, die Gesetze magnetischer Erscheinungen aus Sätzen der Natur-Metaphysik, mithin a priori zu entwickeln. ${ }^{14}$

Wie Schelling hat Eschenmayer die Erkenntnisse und Theorien der sich seit dem Ende des 18. Jahrhunderts zum Teil rapide entwickelnden Naturwissenschaften rezipiert. Bis 1800 wurden viele Entdeckungen gemacht wie die Entdeckung der Oxydation, die die Entwicklung der Chemie als Wissenschaft ermöglichte. Die galvanischen Versuche waren um 1800 Diskussions-gegenstand, es wurde etwa darüber debattiert, inwiefern es sich hier um elektrische, chemische oder magnetische Prozesse handelte. Das Belebende und die immaterielle »Lebenskraft« der Natur wollte man anhand der Versuche beschreiben. ${ }^{15}$

Neben den naturwissenschaftlichen Erkenntnissen war es die philosophische Theorie der Materie, die Eschenmayer (wie Schelling) im Anschluss an Kant beschäftigte. Es galt auf dieser Grundlage eine prinzipielle dynamische Strukturund Vollzugstheorie zu entfalten. Diese sollte nun zugleich die offenen Fragen der Theorie Kants, zuallererst die Frage der spezifischen Qualitäten der Materie, klären können. Im Hinblick auf die Lösung dieser Frage kam es zur ersten

10 Adolph Carl August Eschenmayer, Principia quaedam disciplinae naturali, inprimis chemiae, ex metaphysica naturae substernenda, Tübingen 1796.

11 Vgl., Eschenmayer, Grundriß der Natur-Philosophie, S. X.

12 Vgl., ebd.: »Meine erste spekulative Kost war die kantische Naturmetaphysik, - der erste große Versuch, den Begriff der Materie durch die Kategorien hindurchzuführen und daraus die unveränderlichen Prinzipien für die Natur zu entwikeln. «Marks, S. 9.

13 Ralph Marks, Konzeption einer dynamischen Naturphilosophie bei Schelling und Eschenmayer, München 1982, S. 12.

14 Tübingen 1797 (2. Aufl. 1798).

15 Vgl., Klaus Stein, Naturphilosophie der Frühromantik, Paderborn/ München u.a. 2004, S. 26-39. 
öffentlichen Auseinandersetzung zwischen Schelling und Eschenmayer, die dann zunehmend die methodischen Fragen nach den Bedingungen der Natur und ihrer Erkenntnis sowie auch die prinzipientheoretischen Fragen und Probleme in den Mittelpunkt rückte. Die hier nicht näher zu erläuternde erste Debatte entzündete sich an der Frage, inwiefern die mathematische Konstruktion der Materie in der Lage sei, spezifische Qualität von Materie aus den quantitativen Verhältnissen der beiden Grundkräfte Attraktion und Repulsion bzw. aus den »Intensitätsgraden des Zusammenwirkens ${ }^{16}{ }^{16}$ der beiden zu konstruieren oder abzuleiten. Eschenmayer war der Ansicht, dass dies möglich sei.

Es ging bei der Debatte von Anfang an nicht um ein exotisches Detailproblem der Naturphilosophie, sondern immer auch um die Möglichkeit, Methodik und Reichweite der (Natur-)Philosophie sowie um die prinzipientheoretische Frage nach der ursprünglichen Genese von Differenz aus der Identität und ihrer Beschreibbarkeit sowie um eine grundlegende Theorie der Bestimmtheit. Dieses Themenspektrum wird 1803 und 1804 von der erneuten Diskussion zwischen Schelling und Eschenmayer, welche dann nicht mehr unter naturphilosophischen Vorzeichen steht, wieder im Hinblick auf Schellings Identitätsphilosophie aufgegriffen. Deutlich lassen sich jeweils Kontinuitäten zwischen den beiden Debatten im Hinblick auf die Position Eschenmayers und auch Schellings erkennen.

Worin bestand aber nun die Differenz zwischen Eschenmayer und Schelling in Bezug auf die Frage der spezifischen Qualität von Materie, wie sie bereits vor 1801 deutlich wurde?

Eschenmayers Theorie der Qualität von Körpern geht davon aus, dass Qualität dadurch zustande kommt, dass das Gleichgewicht bzw. die Indifferenz der beiden Grundkräfte der Materie gestört ist, so dass sich die Kräfte gegenseitig begrenzen und Bestimmtheit gesetzt ist. Dieser nicht als ein zeitlicher zu denkende »Akt« der Störung der Indifferenz, der dazu führt, dass beständig in der Materie die ursprüngliche Indifferenz in Differentem reproduziert wird, ist nach Eschenmayer die $\gg$ Potenzierung «. ${ }^{17}$ (Und diese Potenzierung der Indifferenz wird nun auch Eschenmayers Theorie des Übergangs der Philosophie in Nichtphilosophie - von der »Potenz des Ewigen« zur »Potenz des Seligen ${ }^{18}$ - von 1803 und der mit dieser beabsichtigten Antwort auf die Frage der Differenzgenese aus der absoluten Identität Schellings

16 Editorischer Bericht, in: Friedrich Wilhelm Joseph Schelling, Historisch-kritische Ausgabe [im Folgenden AA], Bd. I,10, hg. v. Manfred Durner, Stuttgart 2009, S. 17.

17 Jörg Jantzen, Eschenmayer und Schelling. Die Philosophie in ihrem Übergang zur Nicht-philosophie, in: Religionsphilosophie und spekulative Theologie. Der Streit um die Göttlichen Dinge (1799-1812), hg. v. Walter Jaeschke, Philosophisch-literarische Streitsachen 3, Hamburg 1994, S. 74-97, hier S. 76; vgl. auch Wuttke, Materialien, S. 266.

18 Adolph Carl August Eschenmayer, Die Philosophie in ihrem Uebergang zur Nichtphilosophie, Erlangen 1803, S. 47. 
zugrunde liegen.) - Für Schelling war ein solcher Begriff der Potenzierung im Zusammenhang des Qualitätenproblems undenkbar, aber auch grundsätzlich schien ihm Eschenmayers Theorie den Übergang von Identität zur Differenz nicht angemessen zu bedenken. Schelling hat 1798 im Ersten Entwurf eines Systems der Naturphilosophie ein Modell von »dynamischer Atomistik« entwickelt, das Eschenmayers Theorie zurückwies und von ursprünglichen Qualitäten in der Materie ausging. ${ }^{19}$ Dies erschien Eschenmayer wiederum ein »unbedingter Empirismus « zu sein: "Schelling geht von einem unbedingten Empirismus aus, d. h. über das erste movens der Natur soll innerhalb des Empirismus keine Frage seyn, der Naturphilosoph, so wie er die Natur antrifft, findet sie schon ins Werden gesetzt, und er kann weiter nichts thun, als die jetzt aktive Naturprinzipien in ihrer Thätigkeit zu entwickeln. $\ll^{20}$

Schellings Aufsatz mit dem Titel Ueber den wahren Begriff der Naturphilosophie und die richtige Art, ihre Probleme aufzulösen von 1801, den er als »Anhang" an denjenigen Eschenmayers in seiner Zeitschrift für spekulative Physik abdrucken ließ, ${ }^{21}$ rückte nun wie bereits angedeutet die methodische Frage der Naturphilosophie in den Mittelpunkt. Hier wurden dieselben Themen berührt, die Gegenstand der brieflichen Auseinandersetzung zwischen Schelling und Fichte waren. Schellings Aufsatz ist ein Dokument, das in die Ausarbeitungszeit des Identitätssystems fällt. ${ }^{22}$

In methodischer Hinsicht hat sich Eschenmayer gegenüber Schelling 1801 für eine Beschreibung der Selbstkonstruktion der Natur, wie sie ursprünglich im Bewusstsein gesetzt ist, als Grundlage der Naturphilosophie ausgesprochen. In seinem Aufsatz Spontaneität $=$ Weltseele, der 1801 in Schellings Zeitschrift für spekulative Physik erschien, vertrat er die Ansicht, dass Bewegung und Leben der Natur auf die Einwirkung des Geistes bzw. der Spontaneität zurückzuführen seien. Eine Beschreibung der reinen Selbstkonstruktion der Natur bzw. der absoluten Identität in der Natur, die von dem »Gewusstsein« der Natur abstrahiert, schien ihm das ursprüngliche »Hineintragen« der Spontaneität, des $»$ Prinzip[s] des Werdens $\ll^{23}$ in die Natur zu überspringen, wie er in seinem Aufsatz gegenüber Schelling festhielt. Die Überzeugung, dass die Gesetze der Natur »Modifikationen«" geistiger Gesetze des Subjektes seien, vertrat

19 Vgl., Editorischer Bericht, AA I,10, S. 17f.

20 Vgl., Adolph Carl August Eschenmayer, Spontaneität = Weltseele oder das höchste Princip der Naturphilosophie, in: Zeitschrift für spekulative Physik, hg. v. Friedrich Wilhelm Joseph Schelling, Bd. 2, H. 1, Jena/ Leipzig 1801, S. 1-68 (= AA I,10. S. 345-373, hier: 346).

21 Vgl., Friedrich Wilhelm Joseph Schelling, Anhang zu dem Aufsatz des Herrn Eschenmayer betreffend den wahren Begriff der Naturphilosophie, und die richtige Art ihre Probleme aufzulösen, in: Zeitschrift für spekulative Physik, hg. v. dems., Bd. 2, H. 1, Jena/ Leipzig 1801, S. 109-146 (= AA I,10, S. 85-106).

22 Vgl., Editorischer Bericht, AA I,10, S. 22; 24.

23 Eschenmayer, Spontaneität $=$ Weltseele, S. 22 (= AA I,10, S. 353). 
Eschenmayer auch noch in seinen naturphilosophischen Werken Jahrzehnte später. ${ }^{24}$ Eschenmayers Theorie der Spontaneität in der Natur in seinem Aufsatz von 1801 bedeutet allgemein eine mit Elementen der Fichteschen Philosophie vorgenommene Neuinterpretation und Ausweitung der skizzierten Potenzierungstheorie auf die allgemeine Naturtheorie. Es ist wiederum eine Theorie der »Belebung « von Natur und der Genese von Bestimmtheit in dieser.

In der Auseinandersetzung von 1801 ging es dann, wie Schellings Replik auf Eschenmayers Aufsatz zeigt, ${ }^{25}$ zunehmend um die methodische Grundlage der Naturphilosophie und die Bedeutung der intellektuellen Anschauung, insofern als die Möglichkeit und der Standpunkt der philosophischen Erfassung und Beschreibung der Selbstkonstruktion der Natur (bzw. der absoluten Identität in der Natur) diskutiert wurden. Eschenmayers eigenwillige Theorie der Nichtphilosophie von 1803 wird dann unmittelbar die naturphilosophische Methodenreflexion der Auseinandersetzung von 1801 fortsetzen.

Nachdem Schelling 1801 seine Darstellung meines Systems der Philosophie veröffentlicht hatte, richtete Eschenmayer grundsätzliche Anfragen an dieses. Die Frage der Genese von Differenz und Bestimmtheit wurde von Eschenmayer nun im Hinblick auf die absolute Identität der Darstellung meines Systems der Philosophie erneut an Schelling gerichtet. Eschenmayer bezweifelte, ob es Schelling mit seiner These einer quantitativen Differenz in der absoluten Identität einerseits und eines absolutes Selbsterkennens der absoluten Identität andererseits gelinge, Identität und Differenz sinnvoll zu unterscheiden. Durch die Setzung einer nur quantitativen Differenz in der Identität gebe es letztlich nur bestimmungslose Identität, weil nur quantitative und nicht »wirkliche« Differenz gesetzt ist. Durch den Gedanken des absoluten Selbsterkennens der absoluten Identität werde zwar wirkliche Differenz gesetzt, aber diese sei dann ebenso absolut wie die Identität und »durchbreche diese«: was Schelling offensichtlich gerade vermeiden wolle. ${ }^{26}$ Im Grunde sind damit die Grundlagen des Systems, wie sie Schelling ausarbeitet, und dieses selbst in Zweifel gestellt. Dennoch hat Eschenmayer sich in der Schrift Die Philosophie in ihrem Übergang zur Nichtphilosophie affirmativ auf das Identitätssystem bezogen und beansprucht, das in seiner Sicht mit der absoluten Identität Schellings gegebene Problem durch seine Theorie des Überganges der Philosophie in Nichtphilosophie zu lösen, in die wiederum eine Konzeption von absolutem Selbsterkennen als Selbstdifferenzieren integriert ist. Dabei ging es Eschenmayer aber eben nicht um eine Absicherung und Klärung der Unternehmung »Identitätssystem«, sondern um dessen »Depotenzierung« zugunsten einer über-

24 Vgl., Wuttke, Materialien, S. 263.

25 Vgl., Schelling, Anhang, S. $118 \mathrm{ff}$. (= AA I,10, S. 89ff.)

26 Vgl., Eschenmayer an Schelling am 21.07.1801, in: AA III,2,1, S. 357-363, hier: 357361. 
geordneten Vollendungsgestalt: der Nichtphilosophie. Will Schelling das Identitätssystem in seiner Prinzipientheorie konsistent durchdenken, wie Eschenmayer impliziert, so müsste er dieses zwangsläufig in der Weise Eschenmayers »herabsetzen " (und es damit letztlich verabschieden). Und Eschenmayer legt nahe, dass Schellings dies bereits unwissentlich vollzogen habe. ${ }^{27}$

Entsprechend dem Vorbericht seiner Schrift ist deren Zielrichtung, die Theorie der Nichtphilosophie als eine Reflexion auf die Grenze des Wissens einzuführen bzw. als eine Theorie der Zuordnung von Glauben und Wissen, in der das Wissen bzw. die Philosophie selbst in ihren Voraussetzungen erfasst werden. ${ }^{28}$ Die Theorie des Überganges der Philosophie in Nichtphilosophie führt die bereits angeführten basalen Theorieelemente und Fragen Eschenmayers weiters.

Eschenmayer ging es zunächst um eine »modifizierte« Theorie von intellektueller Anschauung, die seine Theorie des Überganges in methodischer Hinsicht tragen sollte. Die intellektuelle Anschauung ist in Eschenmayers Verständnis von absoluter Identität eine absolute Identität wausser aller Differenz und Indifferenz « ${ }^{29}$ Nicht ist die intellektuelle Anschauung per se ein absolutes Selbsterkennen, denn dieses wäre ein »Selbstdifferentiiren [...], was mit der Integrität der absoluten Identität nicht vereinbar ist « ${ }^{30}$ Für ein solches bräuchte es einen Bestimmungsgrund, der aber nach Eschenmayer niemals in der absoluten Identität mit gesetzt ist. Ein solches absolutes Selbsterkennen bzw. Selbstdifferenzieren ist erst durch den Glauben gesetzt. Die absolute Identität der intellektuellen Anschauung bzw. Vernunft wird erst im »anschauenden « Glauben ein »wirkliches" absolutes Selbsterkennen, womit es aber sogleich wieder über sich hinaus ist. ${ }^{31}$

Eschenmayer kombiniert die intellektuelle Anschauung mit einem (mit dieser freilich nur schwer verträglichen) Element von religiöser Anschauung, das immer schon Bedingung für die Lebendigkeit der intellektuellen Anschauung sein soll und andererseits - gewissermaßen als Anschauen der intellektuellen Anschauung - eine übergeordnete Selbsterfassung (von anderer Qualität) bedeutet, die Ziel der intellektuellen Anschauung ist, zugleich aber im Sinne des Reflexionsmodells ein Herausgehen aus der intellektuellen Anschauung und Begrenzen, Bestimmen und Beleben dieser sein soll. ${ }^{32}$ Ebendiese Begrenzung und Belebung von außerhalb - offenkundig ist es eben die naturphilosophische Theorie der Potenzierung bzw. Spontaneität, die hier zugrunde liegt - bedeutet die Begrenzung des Wissens. Diese Selbsterfassung soll

27 Vgl., etwa Eschenmayer, Uebergang, § 54, S. 46; § 99, S. 104.

$28 \mathrm{Vgl}$., a.a.O., Vorbericht, o.S.

29 A.a.O., § 73, S. 69.

30 Ebd.

31 Vgl., a.a.O., § 60, S. 46.

32 Vgl., a.a.O., § 77, S. 77.

Suplemento 19 (2014) de Contrastes. Revista internacional de filosofía 
zugleich eine höchst wirkliche, (das heißt: empirische und individuelle), sein, ${ }^{33}$ sie ist ein Übergehen des Wissens in (religiöses) Anschauen.

Heterogene Komponenten werden also in Eschenmayers modifizierter Theorie der intellektuellen Anschauung zusammengeführt. Offensichtlich sind es zwei Modelle, die die Theorie der Begrenzung bzw. der Übergehens tragen: Einerseits das $1801 \mathrm{im}$ Brief an Schelling skizzierte Selbsterkennen der absoluten Identität, das nur als Selbstdifferenzieren zu verstehen sei und einen Bestimmungsgrund von außerhalb der absoluten Identität verlange. Dieses Modell von Selbstdifferenzierung gründet auf der Überzeugung Eschenmayers, dass Erkennen immer ein Hinausgehen über das zu Erkennende bedeutet: ${ }^{34}$ So auch das Selbsterkennen. Bestimmtheit bzw. »Gränze«, wie Eschenmayer schreibt, entstehe im Erkennen dadurch, $\gg d a ß$ ich das Verschwinden des zu Begränzenden in dem Hervortreten eines Andern bemerke $\ll .{ }^{35}$ Nur durch den Akt des absoluten Selbsterkennens wird die an sich differenzfreie Identität als Indifferenz zweier Verschiedener, eines Erkennenden und eines Erkannten bzw. als Indifferenz von Natur und Geist gesetzt und in diesem Sinne belebt. Die Vernunft geht so aus sich heraus im absoluten Selbsterkennen, was eben nach Eschenmayer ein Übergehen in den Glauben ist.

Und andererseits steht, wie bereits bemerkt, in Kombination mit dem Modell des absoluten Selbsterkennens, die naturphilosophische Theorie der Potenzierung im Hintergrund, die das Beleben und Stören der absoluten Einheit der absoluten Identität zur Selbstdifferenzierung plausibilisieren soll und die freigesetzte Dynamik und Systematizität erklären soll. Die oben beschriebene Funktion des Potenzierens der Kräfteeinheit bzw. des »Einwirkens der Spontaneität auf die Natur« soll hier entsprechend der Glaube gegenüber der Vernunft erfüllen bzw. die religiöse Nichtphilosophie gegenüber der Philosophie. Durch die Analogie mit diesem Modell soll die Überordnung und die (letztlich mehr behauptete als begründete) qualitative Verschiedenheit des Glaubens gegenüber dem Wissen beschreiben werden. Es soll aber auch die Grenze bzw. die Weise des Bestimmtseins des »Diesseitigen« durch das »Jenseitige« näher erläutert.

Eschenmayer führt aus, dass der Philosophie bzw. der Spekulation die »Postulate « ihrer Konstruktionen unmittelbar durch Offenbarung gegeben sind. ${ }^{36}$ Der Philosoph »selbst steht mithin auf einem ganz andern Standpunkte als das, was er lehrt und mittheilt«. ${ }^{37}$ Die absolute Identität sei zwar die $»$ Basis $\aleph^{38}$ des Philosophierens: Sie vermittle als absolute Identität die Vernunft als

33 Vgl., a.a.O., § 38, S. 30.

34 A.a.O., Vorbericht, o.S.

35 Ebd.

36 Vgl. a.a.O., § 69, S. 61.

37 a.a.O., § 75, S. 73.

38 Ebd. 
Konstruierendes mit der Vernunft als Konstruiertem. Glaube und Offenbarung sind jedoch immer schon die Voraussetzungen der Philosophie. Nur von der skizzierten Basis der Nichtphilosophie aus lassen sich die Theorie des absoluten Selbsterkennens, die Übergangsfrage, der Zusammenhang des Absoluten und Endlichen bzw. der Zusammenhang von Identität und Differenz bzw. von Identität und Selbsterkennen, im Weiteren aber v.a. auch die Frage des Bestimmungsgrundes klären.

Aber auch noch weiter wird das Verhältnis des Absoluten zum Endlichen von Eschenmayer im Anschluss an die Theorie des »Überganges« erläutert. Das im Glauben lebendig gewordene Absolute ermöglicht erst Freiheit in der Welt: $»$ Der göttliche Funke der Freyheit, welcher aus der unsichtbaren Welt sich der unsrigen mittheilt, durchbricht die absolute Identität [...], jetzt erst entsteht mit einem Worte Leben und Weben durch die ganze Sinnen- und intellektuelle Welt «. ${ }^{39}$ Da Schelling ja in der Sicht Eschenmayers die Lebendigkeit des Absoluten nicht aufzuzeigen vermag, so ist damit indirekt gesagt, dass Schelling auch kein Freiheitsverständnis auf dem Boden seines Identitätssystems möglich ist. Dies impliziert auch der Hinweis, dass Schelling die Tugend aus seinem System ausgeschlossen habe. ${ }^{40}$

Eschenmayer beansprucht also mit seiner Theorie der Nichtphilosophie eine konsistente identitätsphilosophische Theorie entfaltet zu haben. Stärker aber wiegt das persönliche Anliegen Eschenmayers, den Glauben nicht nur gegenüber dem Wissen retten zu wollen, sondern als Bedingung und Ziel des Wissens diesem überzuordnen. Die von Eschenmayer gegenüber Schelling immer wieder unterstrichene Unmöglichkeit einer philosophischen Lösung des Problems des Bestimmungsgrundes im Absoluten wird stark funktionalisiert zu diesem Zweck. Die Funktion, die Eschenmayer dem Glauben beimisst, ist groß, der Glaube muss eine gewaltige Last an grundsätzlichen philosophischen Problemen tragen, die er zu lösen beansprucht, wie die Frage nach dem Grund aller Bestimmtheit und der Freiheit sowie die Erklärung des Überganges von Identität zur Differenz. Eschenmayers »Glaube«, »Andacht«, »Ahndung« bleiben jedoch sehr unbestimmt. Dass der Glaube philosophisch nicht näher expliziert werden muss, weil er gerade der Philosophie entzogen ist, ist angesichts seiner großen Bedeutung für Philosophie und Wissen wenig überzeugend. Ihm wird die Lösung komplexer philosophischer Probleme anheimgegeben, sie werden damit aber nicht gelöst, sondern nur an einen Ort verfrachtet, der dem philosophischen Diskurs nicht mehr zugänglich ist. Damit bleiben letztlich doch nur Beteuerungen und das persönliche Anliegen einer Depotenzierung des Wissens zugunsten des Glaubens.

39 a.a.O., § 86, S. 90.

40 Vgl., ebd. 
Am Ende seines Werkes fasst Eschenmayer rückblickend das Anliegen seiner Theorie des »Übergangs « zusammen: »Ich habe, wie ich glaube, der Philosophie nur den Ueberwuchs über ihre eigene Gränzen geschnitten, und sie vermittelst des ewigen Verhältnisses des Glaubens rektificirt. Was ich der Philosophie nahm, das gab ich der Religion wieder [...].. ${ }^{41}$ Schelling wird dann 1804 mit dem Titel seiner Schrift, aber auch direkt am Ende der Einleitung von Philosophie und Religion den Faden wieder aufnehmen und sein »Vorhaben« umsetzen, »diejenigen Gegenstände, welche der Dogmatismus der Religion und die Nichtphilosophie des Glaubens sich zugeeignet haben, der Vernunft und der Philosophie zu vindiciren $\ll$. ${ }^{42}$

\section{DAS IDENTITÄTSSYSTEM UND SCHELLINGS ANTWORT AN ESCHENMAYER}

Schelling rückte in seiner Antwort an Eschenmayer in Philosophie und Religion nicht erneut die Naturphilosophie in den Blickpunkt. Seine Schrift möchte eine ohnehin beabsichtigte, in der vorliegenden Form jedoch unfertige Darstellung der Identitätsphilosophie sein und soll nicht eine Streitschrift allein sein. ${ }^{43}$ In Philosophie und Religion sind es folgende Aspekte, die Schelling vor allem zur Zurückweisung Eschenmayers heranzieht: Die Theorie der intellektuellen Anschauung und das Verständnis von absoluter Identität und ihrem Selbsterkennen, welche Eschenmayer missverstehe, wenn er dieses als ein Selbstdifferenzieren und ein Herausgehen des Absoluten aus sich begreife. Damit verbunden ist eine Reflexion auf das Verhältnis von Absolutem und Endlichen, das heißt auf die Übergangsfrage und auf das Wesen des Endlichen. Der von Eschenmayer diagnostizierte »Ausschluss der Tugend $\aleph^{44}$ ist Anlass zur Präsentation einer (geschichtsphilosophischen) praktischen Philosophie, während Schelling dann Eschenmayers Lehre von der Unsterblichkeit der Seele zurückweist. $^{45}$

Nachfolgend sollen einige Grundgedanken des Identitätssystems skizziert werden, um von dort aus die durch Eschenmayer in Frage stehenden Aspekte in der Sicht Schellings zu rekonstruieren. Die methodische Grundlage der Philosophie soll zunächst in den Blick genommen werden, da sie, wie bereits in der Rekonstruktion von Eschenmayers Theorie der intellektuellen Anschauung von 1803 deutlich wurde, eine Zentralfrage der Auseinandersetzung darstellt. Auch Schelling hat die Theorie der intellektuellen Anschauung und die damit

41 A.a.O., § 100, S. 107.

42 Friedrich Wilhelm Joseph Schelling, Philosophie und Religion, Tübingen $1804=\mathrm{SW}$ I,6, S. 13-70; hier S. 7 = SW I,6, S. 20.

43 Vgl., a.a.O., S. III = SW I,6, S. 13.

44 A.a.O., S. $59=$ SW I,6, S. 53.

45 Vgl., a.a.O., S. 68-74 = SW I,6, S. 60-64. 
verbundene Theorie der absoluten Identität bereits in der Einleitung seiner Schrift als einen entscheidenden Differenzpunkt markiert ${ }^{46}$ und ausgehend von der intellektuellen Anschauung die »Abkunft der endlichen Dinge aus dem Absoluten ${ }^{47}$ in den Blick genommen. In Philosophie und Religion wird jedoch die auf der intellektuellen Anschauung basierende Methodik des Identitätssystems, die Konstruktion, ${ }^{48}$ nicht näher dargelegt, obwohl sie in der Schrift vorausgesetzt ist und die von Schelling seit 1801 präsentierte Philosophieform erst näher zu charakterisieren vermag.

Schellings Methode der Konstruktion möchte Einzelnes im Medium des Absoluten auf die mit ihm gesetzte Idee durchsichtig machen. Die Ideen als bestimmte Einheiten von Besonderem und Allgemeinem, Reellem und Ideellem sollen so dargestellt werden. Das Einzelne soll in Bezug auf das Absolute, in seinem An-sich durchsichtig gemacht werden. Die Konstruktionen beschreiben auf jeder Stufe des Lebens die Ideen, welche drei Momente umfassen, nämlich Allgemeines und Besonderes sowie deren Vermittlung. Dasjenige, was in der Konstruktion demonstriert wird, »ist die absolute Einheit des Endlichen und Unendlichen «; das woran demonstriert wird, »ist eine bestimmte Einheit ${ }^{49}{ }^{9}$ Eine Pointe dieses Verfahrens ist, dass durch dieses die Idee erscheinen soll und die empirische Bestimmtheit, seine kontingente Gestalt, abgeblendet bzw. »zernichtet « wird. Es soll gerade das Besondere als Besonderes am Einzelnen, $»$ in der Entgegensetzung gegen das Allgemeine, vernichtet« werden. ${ }^{50}$

Philosophische Konstruktion leitet damit das Endliche nicht aus dem Absoluten ab, was gegenüber Eschenmayer festzuhalten ist, welcher eine solche Ableitung als Intention des Identitätssystems begreift. Sondern die philosophische Konstruktion vernichtet die Endlichkeit des Einzeldings, um es anschauend auf der eigentlichen Konstruktionsebene darzustellen. Dabei wird die entsprechend der Schwerkraft in der Materietheorie gedachte konstruktive Kraft des Absoluten am Einzelnen dargestellt und zwar so, dass das Absolute darin nur indirekt erscheint. Nur hier, nicht etwa in der empirischen Wirklichkeit, kann nach der Erscheinung oder Realisierung der absoluten Identität im Einzelphänomen gefragt werden. Durch Einführung dieser Unterscheidungen von Ebenen wird die »Zernichtung« des Empirischen an den Dingen zu einer Grundaufgabe der am Absoluten orientierten Philosophie, die

46 Vgl., a.a.O., S. 4-7 = SW I,6. S. 18 f.

47 A.a.O., S. $18=$ SW I,6, S. 28.

48 Vgl., zur Konstruktion: Ueber die Construktion in der Philosophie, in: Kritisches Journal der Philosophie, hg. v. Friedrich Wilhelm Joseph Schelling und Georg Wilhelm Friedrich. Bd. 1. St. 3, Tübingen 1802. S. 26-61 = SW I,5, S. 125-151; Fernere Darstellungen aus dem System der Philosophie, in: Neue Zeitschrift für speculative Physik, Tübingen 1802, Bd. 1, St. 2, S. 3-33 = SW I,4, S. 392-411.

49 A.a.O., S. $5=$ SW I,4, S. 392

50 A.a.O., S. $6=$ SW I,4, S. 393. 
die harsche Zurückweisung aller Standpunkte von Reflexion - wie desjenigen Eschenmayers - nach sich zieht, welche in der Sicht Schellings empirische Reste bzw. endliche Differenzstrukturen in den Standpunkt des Absoluten mit hineinnehmen. Diese »Zernichtung« kehrt dann auch in ähnlicher Weise in der philosophischen Propädeutik und Sittlichkeitslehre wieder.

Die verschiedenen Darstellungen der Identitätsphilosophie ab 1801 kommen darin überein, dass sie das Absolute als absolute Identität fassen, die den Einheitsgrund von Natur und Geist darstellt, der von diesen beiden jedoch unabhängig ist. Damit soll das bisherige System Schellings mit seiner Trennung bzw. Parallelisierung von Transzendental- und Naturphilosophie so weiterentwickelt werden, dass gerade diese Trennung, in der sich die Differenz von Subjekt und Objekt widerspiegelt, überwunden wird. Es sind systematische Absichten, die Schelling in der Vorrede der Darstellung meines Systems der Philosophie anführt. ${ }^{51}$ Es sollen Natur und Geist als qualitativ nicht verschiedene, aber unterscheidbare Einheiten im gemeinsamen, einheitlichen Prinzip begriffen werden. Schelling möchte mit dieser Philosophieform sämtliche gewusste Wirklichkeit in Natur- und Geistsphäre daraufhin durchsichtig machen, inwiefern in ihnen die absolute Identität zur Erscheinung kommt.

Das anhand der Ideen bereits dargelegte, in allen Konstruktionen vorausgesetzte Grundmodell einer Einheit aus zwei Momenten, die durch ein drittes gleichgesetzt sind, gewinnt Schelling aus seiner Theorie der Materie, die eine Weiterentwicklung gegenüber Kants Materietheorie darstellt und für die Herausbildung der Identitätsphilosophie von großer Bedeutung ist. Alles, was ist, entspricht im identitätsphilosophischen Zusammenhang grundsätzlich diesem Muster. Die Materie ist eine Einheit der beiden Grundkräfte »Attractiv-« und »Expansivkraft $\ll^{52}$ (= A und B), wobei Schelling gegenüber Kant die Schwerkraft als dritte Kraft einführt, die die beiden Grundkräfte als gleich und so als seiend in einer synthetischen Einheit setzt, obgleich sie selbst in dieser Funktion als »unmittelbarer Grund von Realität« als »construirende Kraft $\iota^{53}$ nur immanent, das heißt indirekt in der Realität wirklich ist. Die Schwerkraft fungiert als die Einheitsbedingung, als eine indirekt wirkliche Stabilisierung einer in sich dynamischen Einheit. Die Schwerkraft ist so der Repräsentant der absoluten Identität in der Materie, die absolute Identität erscheint in der beschriebenen Weise als eine Kraft, die »auf das Seyn des Products, welches durch $A=B$ bezeichnet wird $\ll,{ }^{54}$ geht.

51 Friedrich Wilhelm Joseph Schelling, Darstellung meines Systems der Philosophie, in: Zeitschrift für spekulative Physik, hg. v. dems., Bd. 2, H. 2, Jena/ Leipzig 1801, Vorerinnerung, S. III-IX (= AA I,10, S. 109-112).

52 Darstellung meines Systems, § 53 Zus. 3, S. $40=$ AA I,10, S. 146.

53 A.a.O., § 54 Erkl., S. $40=$ AA I,10, S. 146.

54 A.a.O., $\S 63$, S. $47=$ AA I,10, S. 152. 
Eschenmayers Theorie der Materie im Aufsatz Spontaneität $=$ Weltseele nimmt gegenüber Schellings Theorie bezeichnenderweise ein viertes Prinzip auf, nämlich die Spontaneität, die erst die Lebendigkeit in der Natur erklären soll: »Es giebt vier Prinzipien: / 1) und 2) Zwei entgegengesetzte Grundkräfte (Mittag und Mitternacht, die zwei Seiten des Dreiecks nach Baader). / 3) Die Schwere (der Niedergang, die einende Basis des Dreiecks). / 4) Der ursprüngliche Trieb (der Aufgang, der pulsirende Zentralpunkt des Dreiecks) Spontaneität $=$ Weltseele $\aleph^{55}$ - Demgegenüber hat das Einzelding der Natur nach Schelling immer schon eine Eigendynamik und muss nicht erst beseelt werden, es ist lebendig durch die zwar selbst immer außerhalb verbleibende Schwerkraft, die aber am Einzelnen als einem bestimmten Produkt der beiden widerstreitenden Grundkräfte für dessen innere Stabilität und die Tendenz des »Gehens auf Sein«, des Setzens in Bestimmtheit steht.

So ähnlich Eschenmayers und Schellings Konzeption auf den ersten Blick erscheinen mögen: Eschenmayer geht letztlich immer von der Spontaneität des Selbstbewusstseins und seiner Struktur aus und lässt so nicht das Selbstbewusstsein von der Natur her entstehen. Insofern haben auch naturphilosophische Strukturen in der Beschreibung von Phänomenen des Geistes eine andere (eher nur illustrierende) Bedeutung. ${ }^{56}$ Und auch grundsätzlich führt dies - bei allen Parallelen zwischen Schelling und Eschenmayer - zu einer anderen Ausarbeitung des Überganges von der Natur zum Geist. Schellings Naturphilosophie hat in der angedeuteten Weise grundlegendere Bedeutung für seine Identitätsphilosophie, gerade auch für die ideelle Philosophie und die Theorie des Absoluten und seiner Erscheinungsweise in der Endlichkeit. Dass die Debatte von 1803 und 1804 nicht erneut zu einer auch naturphilosophischen geworden ist, ist insofern bedauerlich. Die Theorie der Konstruktion ist eine Theorie der Bestimmtheit und möchte das immer schon (im eigentlichen Sinne) Wirkliche am Einzelding, die Idee, darstellen. So geht die Konstruktion Schellings ganz anders mit Bestimmtheit um als Eschenmayers. Das immer schon Wirkliche am Ding wird mit der Idee dargestellt.

Die Konstruktion ist gewissermaßen eine Theorie von intellektueller Anschauung im Vollzug der Philosophie. Insofern unterscheidet sie sich grundlegend von der oben rekonstruierten der oben rekonstruierten »modifizierten«

55 Eschenmayer, Spontaneität $=$ Weltseele, S. 64 = AA I,10, S. 371.

56 Vgl., hierzu etwa die Einführung der Grundkräfte der Materie im Zusammenhang der Darstellung des »Wechselverhältniß[es] « des Selbstbewusstseins in Eschenmayers Dedukzion des lebenden Organism (in: Magazin zur Vervollkommnung der theoretischen und praktischen Heilkunde, Bd. II,3, hg. v. A. Röschlaub, Frankfurt a.M. 1799, S. 329-390, hier S. 372: „Um die Sache deutlicher zu machen, nehme ich einige Beispiele aus der Naturlehre (nicht als ob ich meine Sätze aus dieser begründen wollte, denn es verhält sich umgekehrt, diese Sätze begründen erst die Naturlehre) $\ll$. 
intellektuellen Anschauung Eschenmayers, die seine Theorie des Überganges prägt. Wie gesagt erwähnt Schelling den Begriff »Konstruktion« als methodischen Grundbegriff in Philosophie und Religion nicht. Doch scheint seine Darstellung sowohl der intellektuellen Anschauung als auch der absoluten Identität gerade auf der Grundlage der Theorie der Konstruktion zu erfolgen, ja diese Theorien sind in gewissem Sinne philosophietheoretische Selbstreflexionen. Wie im Vollzug der Philosophie das Unbedingte indirekt erscheint, gilt die Aufmerksamkeit Schellings. Damit soll gegenüber Eschenmayer aufgezeigt werden, dass die Philosophie bei der indirekten Darstellung des Unbedingten nicht von (religiöser) Offenbarung von Evidenz und Bestimmtheit $^{57}$ abhängig ist. So ist auch die Theorie des Absoluten in Philosophie und Religion eine Darstellungstheorie. ${ }^{58}$

Wenn nach Schelling das Absolute als absolute Identität ist, dann sind auch die Form bzw. die Ideen in der konstruierten Reinstruktur absolut, insofern sie die Darstellungsmedien des Absoluten sind und auf das Absolute beziehbar sein müssen. Die Form der Bestimmtheit der Ideen soll eine positive, ihre Realität eine ideell-reelle Normativität sein (welche sich freilich wiederum nur an einem konkreten Einzelnen darstellen kann). Die Einheit von Absolutem und seiner Darstellung im Vollzug beschreibt Schelling als ein »Selbsterkennen $\ll::^{59}$ und dies unter Abgrenzung von Eschenmayer, der mithilfe seiner Theorie der Reflexion und des Selbstdifferenzierens das Darstellungsmedium des Absoluten und die Bestimmtheit erst erzeugen muss, wie wir sahen.

Schelling ist mit seiner Identitätsphilosophie (anders als Eschenmayer) an der Darstellung der Selbsterfassung des Absoluten orientiert. Die Geschichte des Absoluten in der Geschichte zu beschreiben bzw. seine Wirklichkeit in der Selbstbestimmung zu erfassen, ist nach Schelling Thema der Religion, die hier in einem ganz anderen Horizont steht als bei Eschenmayer.

Nach Schelling ist die Religion auf dem Boden der Philosophie und durch diese beschreib- und konstruierbar. Philosophie und Religion haben, wie

57 Vgl., Schelling, Philosophie und Religion, S. 15f.: »Inwiefern diese Anschauung nicht gleich der irgend einer geometrischen Figur auf allgemeingeltende Art gegeben werden kann, sondern jeder Seele, wie die Anschauung des Lichts jedem Auge, insbesondere eigenthümlich ist, so ist hier allerdings eine bloß individuelle, aber in dieser Individualität doch eben so allgemeingültige Offenbarung, als es für den empirischen Sinn das Licht ist, und dieß könnte der Punct seyn, durch welchen auch Eschenmayers Andeutungen sich mit den Behauptungen der Philosophie, in der beyderseitigen weitern Entwicklung vereinigen lassen möchten.« (= SW I,6, S. 26)

58 Vgl., Christian Danz, Die Begründung des Gottesgedankens in Philosophie und Religion. Anmerkungen zu den problemgeschichtlichen und systematischen Voraussetzungen von Schellings philosophischer Theologie, in: F.W.J. Schelling, Philosophie und Religion, hg. v. Alfred Denker u. Holger Zaborowski, Interpretationen und Quellen 1, München 2008, S. 155-168, hier S. 159ff.

59 Schelling, Philosophie und Religion, S. $24=$ SW I,6, S. 31. 
Schelling anhand der Mysterienreligion darlegt, dasselbe Erkenntnisprinzip. ${ }^{60}$ Die intellektuelle Anschauung wird also von Schelling nicht um ein übergeordnetes »religiöses« Moment ergänzt wie bei Eschenmayer. So ist in Schellings Theorie in methodisch-prinzipieller Hinsicht ein Konkurrenzverhältnis von Philosophie und Religion oder eine Überbietungsfigur wie in Eschenmayers Theorie ausgeschlossen. Auch haben nach Schelling Philosophie und Religion dasselbe Absolute zum Thema. ${ }^{61}$ Was sie unterscheidet, ist eine unterschiedliche Perspektive auf das Absolute: Die Philosophie stellt das Absolute in seiner Selbstkonstruktion, in seiner unbedingten Selbsterfassung in den Ideen der Natur und Geschichte dar. Erst in der Geschichte erscheint das Absolute als solches. ${ }^{62}$

Die Religion hat die Wirklichkeit der absoluten Selbstbestimmung und der Sittlichkeit zum Thema, in der sich das Absolute als solches zeigt. Religion ist der Vollzug und die Beschreibung der absoluten Selbstbestimmung am Ort des konkreten Individuums. Sie ist so ein spezifisches Wissen bzw. Anschauen und Darstellen des Erscheinens des Absoluten in der Endlichkeit. ${ }^{63}$ Die Wirklichkeit des Vollzuges der Absoluten Selbstbestimmung und damit des Absoluten am Ort des Individuums kann nur von der Religion als einer »Freiheits- und Endlich-keitshermeneutik « erfasst und beschrieben werden, so dass die Philosophie insofern die Religion in ihrer Eigenbedeutung anerkennt.

Die Attraktivität der Schellingschen Konzeption von Religion gegenüber derjenigen Eschenmayers liegt gerade darin, dass Schelling mit der Möglichkeit der (philosophischen) Explizierbarkeit von Religion bei gleichzeitiger Anerkennung ihres Eigenwertes für das Individuum und seinen Selbstvollzug rechnet. Eschenmayer dagegen führt eine Überbietungsfigur ein, aus der folgt, dass die Religion selbst nicht dem Wissen zugänglich ist: obgleich sie gerade für das Wissen und die Philosophie die Lösung ihrer prinzipiellen Probleme leisten soll.

PATRICK LEISTNER is research associate at the »Institut für Systematische Theologie und Religionswissenschaft « of the »Evangelisch-Theologische Fakultät der Universität Wien «.

Area of research:

Deutsche Idealismus, Schelling, Theologie und Religionswissenschaft.

Recent publications:

60 Vgl., a.a.O., S. 4-6 = SW I,6, S. 18-20.

61 Vgl., a.a.O., S. $3 ; 77-80=$ SW I,6, S. 17; 67-70.

62 Vgl., etwa Schelling, Fernere Darstellungen, Bd. 1, St. 2, S. 47 = SW I,4, S. 421.

63 Schelling, Philosophie und Religion, S. 75-82 = SW I,6, S. 65-70. 
At the moment he works on the edition of volume I,14 of the Historical-Critical Edition of F.W.J. Schelling.

e-mail: patrick.leistner@univie.ac.at 